\title{
Photocatalytic Water Oxidation with a CoFe Prussian Blue Analogue-Layered Niobate Hybrid Material
}

\author{
Sina Sadigh Akbari, Ugur Unal,* and Ferdi Karadas*
}

Cite This: ACS Appl. Energy Mater. 2021, 4, 12383-12390

Read Online

ABSTRACT: A semiconductor-catalyst hybrid assembly for photocatalytic water oxidation was obtained by preparing $\mathrm{CoFe}$ Prussian blue particles on Dion-Jacobson type niobate nanosheets, which produces a $\mathrm{p}-\mathrm{n}$ junction, as evidenced by the MottSchottky plot. The hybrid material with a precious-metal-free cocatalyst exhibits an enhanced photocatalytic activity $(89.5 \mu \mathrm{mol}$ $\mathrm{g}^{-1} \mathrm{~h}^{-1}$ ) in the presence of $\mathrm{S}_{2} \mathrm{O}_{8}{ }^{2-}$ as the electron scavenger. XPS, infrared, XRD, TEM, and SEM studies performed on both pristine and postcatalytic samples indicate that the hybrid assembly exhibits a proper band energy alignment for the photocatalytic water oxidation process and it is stable throughout a $12 \mathrm{~h}$ photocatalytic study.

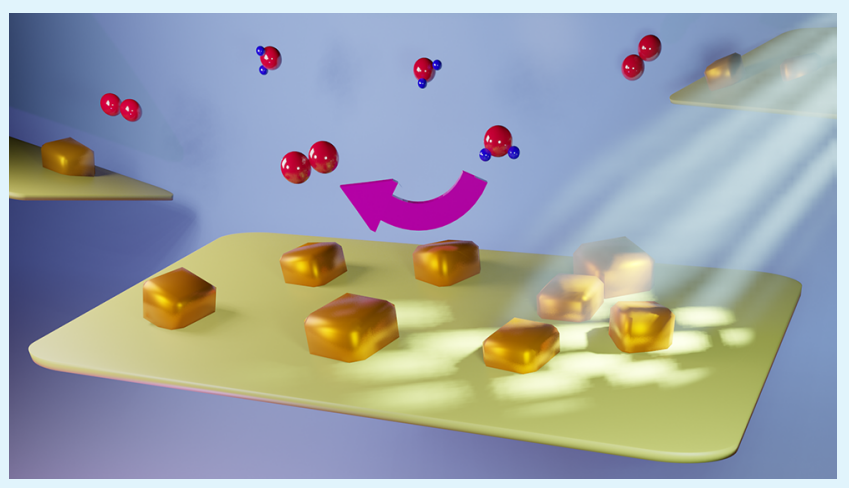

KEYWORDS: water splitting, photocatalytic water oxidation, calcium niobate nanosheets, Co-Fe Prussian blue analogue

\section{INTRODUCTION}

Photocatalytic water splitting relies mainly on an oxide-based semiconductor with proper energy levels with respect to those of water oxidation and reduction reactions. Among them, twodimensional (2D) layered perovskites have attracted attention, since their electronic properties and surface areas can be modified simply through ion exchange, intercalation, and reconstruction of the layered structure after exfoliation. ${ }^{1-3}$ One of the typical examples of layered oxides, $\mathrm{KCa}_{2} \mathrm{Nb}_{3} \mathrm{O}_{10}$, exhibits a Dion-Jacobson type layered perovskite structure, in which negatively charged metal oxide sheets $\left(\mathrm{Ca}_{2} \mathrm{Nb}_{3} \mathrm{O}_{10}{ }^{-}\right)$are separated by potassium ions. ${ }^{3}$ These perovskite sheets can easily be obtained after proton exchange and exfoliation reactions. The dimensionality of the structure is reduced from a bulk species to $2 \mathrm{D}$ nanosheets with the exfoliation process, which provides a high surface area, improved electrical properties, and enhanced photocatalytic activities. ${ }^{4-6}$ In addition, 2D nanosheets of exfoliated layered perovskites can be used as building blocks to design and develop new materials for various applications. ${ }^{3}$ Dye molecules, oxides, quantum dots, and even metallic nanostructures have previously been incorporated into the layered niobates using the aforementioned method. ${ }^{7-14}$ This approach holds the potential not only to inhibit the recombination of electron-hole pairs by stimulating charge separation but also to develop semiconductor-catalyst hybrid assemblies.

$\mathrm{CoFe}$ Prussian blue analogues have recently emerged as potential water oxidation catalysts. ${ }^{15-18}$ The metal ions that are connected through a cyanide group yield a rigid and robust structure, which is stable even under harsh electrocatalytic and photocatalytic conditions over a wide $\mathrm{pH}$ range, $\mathrm{pH} 1-13 .{ }^{19}$ The iron sites in a CoFe Prussian blue (CoFe-PB) structure are catalytically inactive due to the lack of an available coordination site for catalytic activity. ${ }^{20,21}$ On the other hand, octahedral cobalt sites, either on the surface or in the interstitial vacancies that are created due to charge balance, complete their coordination spheres with water molecules, which make them ideal catalytic sites for water oxidation. These cobalt sites can be oxidized to higher oxidation states under a relatively low overpotential to activate them for the catalytic water oxidation process. It is also possible to promote an efficient hole transfer from a semiconductor and/or a photosensitizer to the cobalt site for photocatalytic and photoelectrochemical water oxidation..$^{22-25}$ In such an architecture, the valence band of the semiconductor is expected to be lower than the HOMO level of the Prussian blue structure for an efficient charge transfer. As wide-band-gap semiconductors, layered niobates satisfy this condition.

Previous studies on both layered niobates and Prussian blue analogues suggest that they could collaborate for a photocatalytic water oxidation process due to their proper energy level alignments. ${ }^{26,27}$ While bare layered niobates are suitable

Received: July 23, 2021

Accepted: October 12, 2021

Published: October 28, 2021 
candidates for water reduction, they need to be either sensitized or coupled with a catalyst for the water oxidation reaction. ${ }^{28}$ It has been claimed in several publications that the exfoliation/restacking route is essential for oxygen evolution from niobate sheets. ${ }^{28-30}$ The restacking route acquires a larger surface area for high specific activity and is beneficial for the accessibility of reactant molecules such as water to reaction sites on individual nanosheets. Platinum nanoparticles, $3 \mathrm{~d}$ metal oxides, and ruthenium tris(bipyridyl) complexes have previously been intercalated into niobate oxide sheets. ${ }^{13,14,31}$ On the other hand, CoFe Prussian blue analogues have previously been combined with photosensitizers and/or semiconductors for the light-driven water oxidation process. For the development of Prussian blue based photocatalytic hybrid assemblies, a variety of components have been utilized ranging from ruthenium photosensitizers to porphyrin derivatives, oxide-based semiconductors, and layered double hydroxides. ${ }^{32-37}$ Herein, we combine a layered niobate and a CoFe Prussian blue structure to develop a semiconductorcatalyst hybrid assembly for the photocatalytic water oxidation process.

\section{EXPERIMENTAL SECTION}

Synthesis of Layered $\mathrm{KCa}_{2} \mathrm{Nb}_{3} \mathrm{O}_{10}$. A Dion-Jacobson phase $\mathrm{KCa}_{2} \mathrm{Nb}_{3} \mathrm{O}_{10}$ layered structure was synthesized by a complex polymerization method. ${ }^{31} \mathrm{NbCl}_{5}$ (3.06 g, Alfa Aesar, 99.999\%), $\mathrm{CaCO}_{3}\left(0.77 \mathrm{~g}\right.$, Alfa Aesar, 99.9\%), $\mathrm{K}_{2} \mathrm{CO}_{3}(0.30 \mathrm{~g}$, anhydrous, Alfa Aesar, 99\%), anhydrous citric acid (CA; 21.83 g, Sigma-Aldrich, ACS Reagent 99.5\%), and ethylene glycol (EG; $25.40 \mathrm{~mL}$, Sigma-Aldrich, $>99 \%$ ) were used as precursor materials. In a typical synthesis, $\mathrm{NbCl}_{5}$ was dissolved in $113.43 \mathrm{~mL}$ of methanol (Merck, anhydrous $99.8 \%$ ). Then, appropriate amounts of $\mathrm{CaCO}_{3}$ and $\mathrm{K}_{2} \mathrm{CO}_{3}$ were added to the solution in a ratio that sustained charge neutrality with continuous stirring. Anhydrous citric acid was used as a chelating agent so that $\left[\mathrm{NbCl}_{5}\right] /[\mathrm{CA}]$ ratio was 0.1 . Moreover, ethylene glycol was in a $[\mathrm{CA}] /[\mathrm{EG}]$ ratio of 4 added to the mixture. An excess of $\mathrm{K}_{2} \mathrm{CO}_{3}(10$ $\mathrm{mol} \%$ of $\mathrm{K}$ ) was added to the solution to compensate for volatilization in the calcination step. The reaction mixture was heated at $\sim 100{ }^{\circ} \mathrm{C}$ for $6-8 \mathrm{~h}$ on a hot plate stirrer. The as-obtained transparent solution was heated to $\sim 300-350{ }^{\circ} \mathrm{C}$ to promote polyesterification between EG and $\mathrm{CA}$ in a heating mantle. The mixture yielded a glassy resin first, and a black powder was obtained at the end of the carbonization reaction. The black powder was calcined at $650{ }^{\circ} \mathrm{C}$ for $6 \mathrm{~h}$ in an $\mathrm{Al}_{2} \mathrm{O}_{3}$ crucible to remove the residual organic content. The obtained white precursor was then further calcined at $800-1200{ }^{\circ} \mathrm{C}$ for $2-5 \mathrm{~h}$ in an $\mathrm{Al}_{2} \mathrm{O}_{3}$ crucible.

Preparation of the Proton-Exchanged Powders. Proton exchange was carried out in an aqueous nitric acid $(6 \mathrm{M})$ at room temperature for 3 days. The products were collected by vacuum filtration, washed extensively with double-distilled $\mathrm{H}_{2} \mathrm{O}$, and dried at $80{ }^{\circ} \mathrm{C}$ overnight.

Preparation of the Nanosheets. Exfoliation of the as-prepared proton-exchanged materials was performed using aqueous tetra- $n$ butylammonium hydroxide (TBA; Aldrich, 40 wt $\%$ in $\mathrm{H}_{2} \mathrm{O}$ ) at room temperature as reported by Maeda et al. ${ }^{31}$ The powder was shaken in an aqueous solution containing TBA for 1 week with the molar ratio $[\mathrm{TBA}] /\left[\mathrm{H}^{+}\right]=1$. The reaction was performed on a shaker for 7 days. Final nanosheet solutions were obtained by storing the supernatant after centrifugation of the solutions at $3000 \mathrm{rpm}$ for $30 \mathrm{~min}$.

Preparation of Niobate/PB Hybrid Assembly. For the preparation of the hybrid structure, $0.18 \mathrm{mmol}$ of $\mathrm{Co}\left(\mathrm{NO}_{3}\right)_{2} \cdot 3 \mathrm{H}_{2} \mathrm{O}$ (Alfa Aesar, 99.9\%) was dissolved in $10 \mathrm{~mL}$ of deionized water, added dropwise to an exfoliated $\mathrm{Ca}_{2} \mathrm{Nb}_{3} \mathrm{O}_{10}{ }^{-}$solution at room temperature, and stirred for $1 \mathrm{~h}$. The $\mathrm{Co}^{2+} /\left[\mathrm{Ca}_{2} \mathrm{Nb}_{3} \mathrm{O}_{10}{ }^{-}\right]$precipitate was collected by centrifugation and washed with water. The obtained pinkish solid was dispersed in deionized water and sonicated for $20 \mathrm{~min}$. Subsequently, an aqueous solution of $\mathrm{K}_{3}\left[\mathrm{Fe}(\mathrm{CN})_{6}\right](0.12 \mathrm{mmol}$ in
$10 \mathrm{~mL}$ of water, Sigma-Aldrich, $99.0 \%$ ) was added dropwise to the above solution and stirred for $18 \mathrm{~h}$. The final precipitate was separated by centrifugation, rinsed with deionized water, and finally dried at 75 ${ }^{\circ} \mathrm{C}$.

Characterization. Powder X-ray diffraction patterns of samples were obtained on a Pananalytical X'PertPro multipurpose X-ray diffractometer (MPD) with $\mathrm{Cu} \mathrm{K} \alpha \mathrm{X}$-ray radiation $(\lambda=1.5418 \AA)$. The diffraction patterns were recorded within the range of $3-80^{\circ}$ with a scan step of 0.04. Fourier transform infrared (FT-IR) spectra were recorded with a Bruker Alpha Platinum-ATR spectrometer with $4 \mathrm{~cm}^{-1}$ resolution in a wavenumber range between 4000 and 400 $\mathrm{cm}^{-1}$. The morphology and chemical composition of the samples were examined by a scanning electron microscope (SEM, FEI-Quanta 200 FEG ESEM) with an energy dispersive X-ray spectrometer (EDS) analyzer. Transmission electron microscopy (TEM) imaging was carried out at $300 \mathrm{kV}$ using an FEI Technai G2 F30 transmission electron microscope. Optical absorption properties of the samples were studied via diffuse reflectance UV-A-vis (DR-UV-A-VIS) spectroscopy using a Cary 5000 UV-A-vis-NIR spectrometer equipped with a Varian Cary 2500 internal diffuse reflectance (DR) accessory. X-ray Photoelectron (XPS) measurements were performed by using a Thermo Scientific K-Alpha X-ray photoelectron spectrometer system equipped with an $\mathrm{Al} \mathrm{K} \alpha$ microfocused monochromator source $(1486.6 \mathrm{eV})$ operating at $400 \mu \mathrm{m}$ spot size and accompanied by a flood gun for charge neutralization.

Photocatalytic $\mathrm{O}_{2}$ Evolution. The photocatalytic experiments were carried out in a $16 \mathrm{~mL}$ Pyrex reactor at room temperature. A 5 $\mathrm{mg}$ portion of the catalyst was suspended in $10 \mathrm{~mL}$ of deionized water containing $5 \mathrm{mM} \mathrm{Na}_{2} \mathrm{~S}_{2} \mathrm{O}_{8}$ as a sacrificial agent. The reaction solution was deaerated by bubbling $\mathrm{N}_{2}$ gas for 30 min before light irradiation. A Xe lamp ( $300 \mathrm{~W}$; AM 1.5 global filter) was used as a light source. The amount of generated $\mathrm{O}_{2}$ was determined at $1 \mathrm{~h}$ intervals by injecting $100 \mu \mathrm{L}$ of the reactor headspace gas into a gas chromatograph (Agilent 7820A GC, with molecular sieves column and a TCD detector). Ar was used as the carrier gas. Two rubber septa were used to seal the reactor, where the space between them was purged with $\mathrm{N}_{2}$ gas to avoid a possible $\mathrm{O}_{2}$ leak during the measurements.

Photoelectrochemical Measurements. (Photo)electrochemical measurements were performed by using a Gamry Instruments Interface 1000 potentiostat/galvanostat. A standard three-electrode cell was used, with $\mathrm{Ag} / \mathrm{AgCl}(3.5 \mathrm{M} \mathrm{KCl})$ as the reference electrode, a Pt mesh as the counter electrode, and FTO $(1 \times 2 \mathrm{~cm} ; 2 \mathrm{~mm}$ slides with $7 \Omega \mathrm{sq}^{-1}$ surface resistivity and $\sim 80 \%$ transmittance) as the working electrode. Before electrode preparation, the FTO slides were washed by ultrasonication for $10 \mathrm{~min}$ each in basic soapy solution, deionized water, and isopropanol and then annealed at $300{ }^{\circ} \mathrm{C}$ for 30 $\mathrm{min}$. The photoanodes were prepared by a drop-casting method. A suspension of the photocatalyst powder $(2 \mathrm{mg})$ and methanol $(200$ $\mu \mathrm{L})$ was prepared by ultrasonication for $30 \mathrm{~min}$. Subsequently, $30 \mu \mathrm{L}$ of the suspension was dropped uniformly on the FTO slide $\left(1 \mathrm{~cm}^{2}\right)$, and dried at $75{ }^{\circ} \mathrm{C}$. The $\mathrm{KCa}_{2} \mathrm{Nb}_{3} \mathrm{O}_{10}$-containing electrode was annealed at $300{ }^{\circ} \mathrm{C}$ for $30 \mathrm{~min}$. All (photo)electrochemical measurements were performed in $0.1 \mathrm{M}$ phosphate buffer solution (PBS; pH 7) containing $0.5 \mathrm{M} \mathrm{Na}_{2} \mathrm{SO}_{4}$ at room temperature. Prior to each experiment, the electrolyte solution was bubbled with $\mathrm{N}_{2}$ gas for $15 \mathrm{~min}$ to remove dissolved $\mathrm{O}_{2}$. Cyclic voltammetry (CV) measurements were accomplished under dark conditions and a scan rate of $50 \mathrm{mV} \mathrm{s}^{-1}$. Chronoamperometry (CA) was measured under chopped illumination at a fixed potential of $1 \mathrm{~V}$ (vs NHE). A MottSchottky analysis was performed with the $1 \mathrm{kHz}$ frequency in the absence of light.

\section{RESULTS AND DISCUSSION}

$\mathrm{KCa}_{2} \mathrm{Nb}_{3} \mathrm{O}_{10}$ compound was synthesized and exfoliated according to the procedures reported in the literature. ${ }^{31,38}$ The exfoliated calcium niobate nanosheets were restacked by cobalt ions and subsequently reacted with a solution of $\left[\mathrm{Fe}(\mathrm{CN})_{6}\right]^{3-}$ to afford a hybrid assembly (abbreviated from 


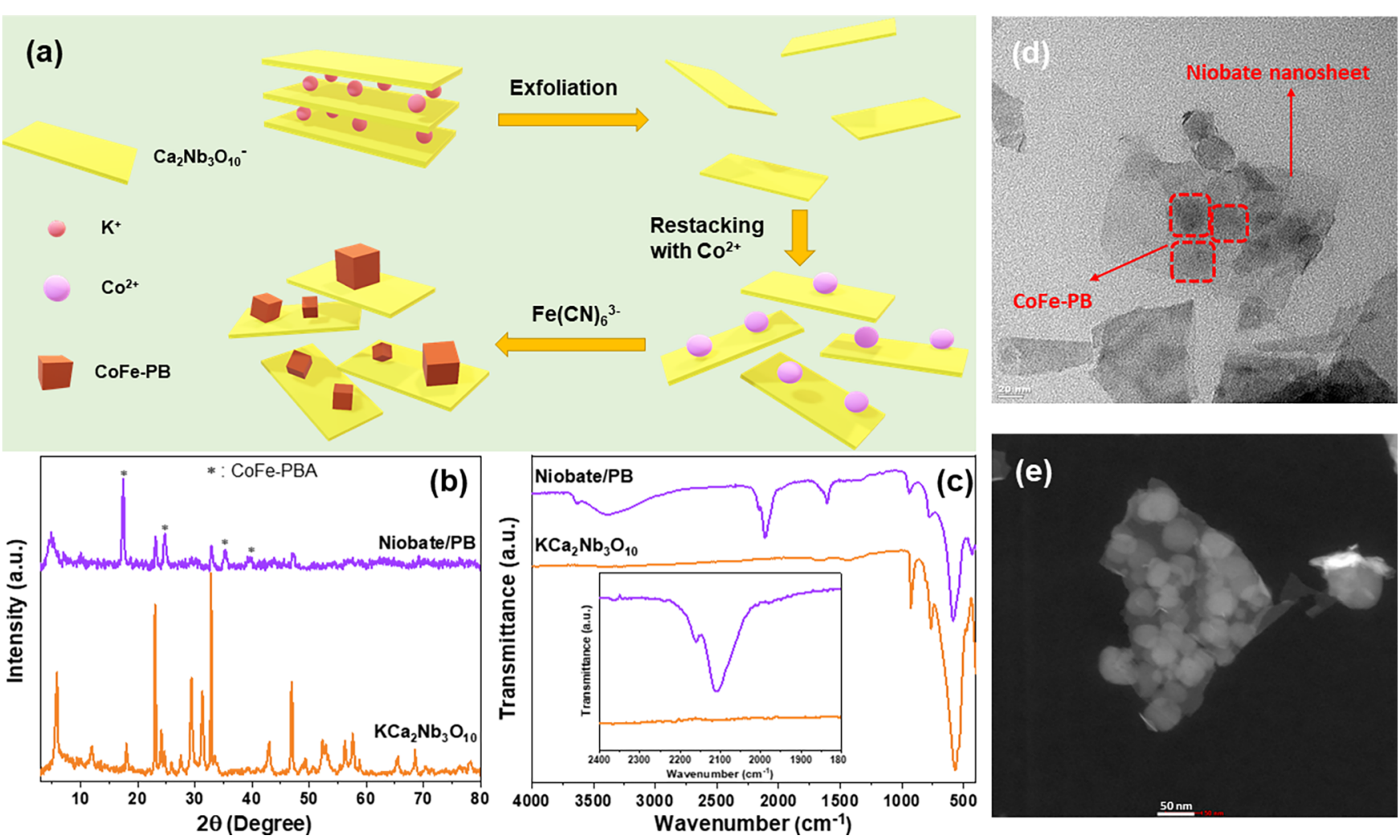

Figure 1. (a) Schematic representation for the in situ synthesis of CoFe-PB on exfoliated $\mathrm{Ca}_{2} \mathrm{Nb}_{3} \mathrm{O}_{10}{ }^{-}$nanosheets at room temperature. (b) XRD patterns of $\mathrm{KCa}_{2} \mathrm{Nb}_{3} \mathrm{O}_{10}$ and Niobate/PB samples. (c) ATR-FTIR spectra of $\mathrm{KCa}_{2} \mathrm{Nb}_{3} \mathrm{O}_{10}$ and Niobate/PB samples. Inset: the cyanide stretching mode. (d) TEM image of the Niobate/PB sample. (e) High-angle annular dark field (HAADF) image of the the Niobate/PB sample.
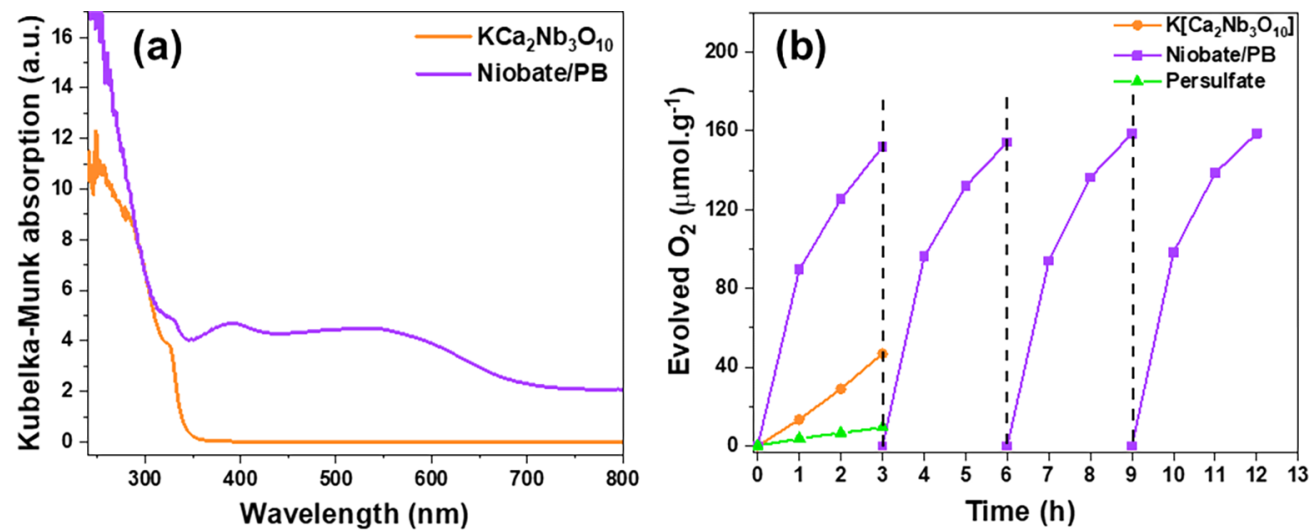

Figure 2. (a) Diffuse reflectance UV-vis spectra (plotted as the Kubelka-Munk function of the reflectance, $R$ ) of $\mathrm{KCa}_{2} \mathrm{Nb}_{3} \mathrm{O}_{10}$ and $\mathrm{Niobate} / \mathrm{PB}$ samples. (b) Photocatalytic oxygen evolution activities of persulfate, $\mathrm{KCa}_{2} \mathrm{Nb}_{3} \mathrm{O}_{10}$, and Niobate/PB samples $\left(\mu\right.$ mol of generated $\mathrm{O}_{2} / \mathrm{g}$ of catalyst) in water containing $5 \mathrm{mg}$ of the catalyst and $5 \mathrm{mM} \mathrm{Na}_{2} \mathrm{~S}_{2} \mathrm{O}_{8}$ as the sacrificial agent under UV-vis irradiation ( $300 \mathrm{~W} \mathrm{Xe} \mathrm{lamp).} \mathrm{After} \mathrm{each} \mathrm{cycle,} 5$ $\mathrm{mM} \mathrm{Na} \mathrm{S}_{2} \mathrm{O}_{8}$ was added to the reaction solution.

now on as Niobate/PB), which is composed of niobate nanosheets and uniform CoFe-PB cubic structures (Figure 1a). Figure $1 \mathrm{~b}$ shows the $\mathrm{X}$-ray diffraction (XRD) patterns of the pristine $\mathrm{KCa}_{2} \mathrm{Nb}_{3} \mathrm{O}_{10}$ and the Niobate/PB samples. The XRD pattern of $\mathrm{KCa}_{2} \mathrm{Nb}_{3} \mathrm{O}_{10}$ exhibits no impurity phase and shows that the layered structure was successfully prepared. The combination of niobate sheets with $\mathrm{PB}$ results in a shift of the (020) peak of the layered niobate structure to $4.8^{\circ}$, which corresponds to an interlayer distance of around 18.2 A. Other peaks at $2 \theta=23.09,32.88$, and $47.14^{\circ}$ belong to the $(110)$, (112), and (040) lattice planes, respectively. The loss of other peaks belonging to the layered $\mathrm{KCa}_{2} \mathrm{Nb}_{3} \mathrm{O}_{10}$ structure and the broad peak at $2 \theta=4.8^{\circ}$ show that the exfoliation/restacking process and formation of $\mathrm{PB}$ arrange $\mathrm{Ca}_{2} \mathrm{Nb}_{3} \mathrm{O}_{10}{ }^{-}$nanosheets in a stratified structure. The diffraction peaks on the diffraction pattern of the hybrid structure at $17.5,24.8,35.2$, and $39.5^{\circ}$ correspond to (200), (220), (400), and (420) lattice planes of a cubic Prussian blue structure. The XRD pattern of Niobate/ $\mathrm{PB}$ thus indicates that the $\mathrm{PB}$ structure was prepared successfully on the niobate nanosheets. ${ }^{16}$

In the Fourier transform infrared (FTIR) spectrum of Niobate/PB, the strong band at $585 \mathrm{~cm}^{-1}$ can be assigned to the asymmetric stretching vibration of central $\mathrm{Nb}-\mathrm{O}$ bonds in the $\mathrm{NbO}_{6}$ octahedron of the perovskite layer. The asymmetric 

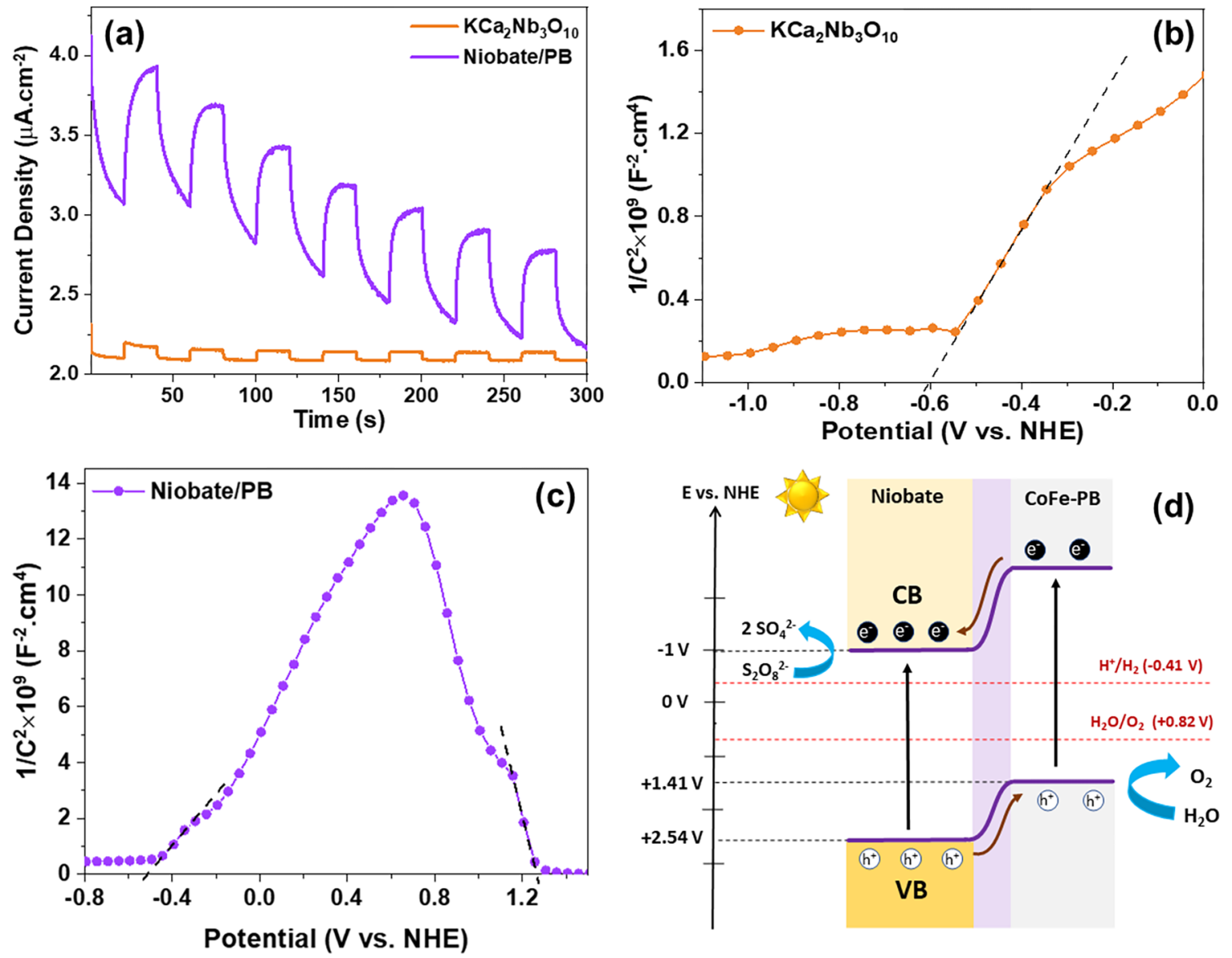

Figure 3. (a) Transient photocurrent responses of $\mathrm{KCa}_{2} \mathrm{Nb}_{3} \mathrm{O}_{10}$ and Niobate/PB samples in $0.1 \mathrm{M} \mathrm{PBS}$ (pH 7) containing $0.5 \mathrm{M} \mathrm{Na}_{2} \mathrm{SO}_{4}$ under UV-vis irradiation ( $300 \mathrm{~W}$ Xe lamp). Mott-Schottky plots of (b) $\mathrm{KCa}_{2} \mathrm{Nb}_{3} \mathrm{O}_{10}$ and (c) Niobate/PB samples. (d) Schematic representation of the Niobate/PB $\mathrm{p}-\mathrm{n}$ junction and charge-transfer mechanism under light irradiation.

stretching vibration of the bridge $\mathrm{Nb}-\mathrm{O}$ in the terminal $\mathrm{NbO}_{6}$ group results in a peak at $778 \mathrm{~cm}^{-1}$, whereas the peak at 939 $\mathrm{cm}^{-1}$ can be assigned to the stretching vibration of the terminal $\mathrm{Nb}-\mathrm{O} .^{39,40}$ Once the niobate nanosheets are coupled to $\mathrm{PB}$ particles, the intensity of the band at $939 \mathrm{~cm}^{-1}$ decreases, which could be attributed to the interaction of terminal $\mathrm{Nb}-\mathrm{O}$ bonds in the perovskite layer with PB structures. The broad band at $3386 \mathrm{~cm}^{-1}$ and the sharp band at $3641 \mathrm{~cm}^{-1}$ are attributed to the $\mathrm{OH}$ stretching of water molecules on the surface of PB particles. Another sharp band at $1606 \mathrm{~cm}^{-1}$ can be assigned to the $\mathrm{H}-\mathrm{O}-\mathrm{H}$ bending mode of water molecules inside the Prussian blue structure. ${ }^{41}$ The two bands at 2110 and $2162 \mathrm{~cm}^{-1}$ are attributed to $\mathrm{Co}$ (III)-NC-Fe(II) and $\mathrm{Co}(\mathrm{II})-\mathrm{NC}-\mathrm{Fe}$ (III) coordination modes, respectively (Figure 1c). ${ }^{42}$

The structure and morphology of the samples were also studied by scanning electron microscopy (SEM) and transmission electron microscopy (TEM) images. SEM images reveal the presence of a platelike layered structure of calcium niobate nanosheets in both samples (Figure S1a-f). A TEM analysis of the Niobate/PB sample indicates the formation of a cubic Prussian blue structure with a size of $20-50 \mathrm{~nm}$ on the niobate layers (Figure 1d,e and Figure S2). The Co:Fe:Nb atomic ratio for the hybrid sample is found to be around 5:3.5:4.2 by an energy-dispersive X-ray spectroscopy (EDS) analysis (Figure S3). EDS elemental mapping of the Niobate/ $\mathrm{PB}$ sample reveals that the $\mathrm{PB}$ structure is well-dispersed on calcium niobate layers to form a uniform hybrid assembly (Figure S4).

The optical properties of $\mathrm{KCa}_{2} \mathrm{Nb}_{3} \mathrm{O}_{10}$ and Niobate/PB samples were studied with $\mathrm{UV}$-vis diffuse reflectance spectroscopy (Figure 2a). The characteristic absorption band of $\mathrm{KCa}_{2} \mathrm{Nb}_{3} \mathrm{O}_{10}$ extends up to $350 \mathrm{~nm}$, which is associated with the electronic transition from the valence band consisting of the $\mathrm{O} 2 \mathrm{p}$ orbitals to the conduction band consisting of empty $\mathrm{Nb} 4 \mathrm{~d}$ orbitals. ${ }^{30}$ On the other hand, the Niobate/PB sample exhibits a broad background absorption in the $350-800 \mathrm{~nm}$ region due to the electronic interaction between the calcium niobate nanosheets and $\mathrm{PB}$ particles. This can be confirmed by the difference in the color of the white bare niobate nanosheets and the dark purple hybrid sample. Two broad absorption regions in the $350-800 \mathrm{~nm}$ range illustrate the characteristic charge transfer in CoFe-PB structures and proves that the hybrid system is active in the visible region. ${ }^{43}$ The inherent band structure of the niobate layers, however, remains unchanged in the hybrid structure, since the crystal and chemical structures of the niobate layers are not modified.

The photocatalytic oxygen evolution performance of $\mathrm{KCa}_{2} \mathrm{Nb}_{3} \mathrm{O}_{10}$ and PB-modified calcium niobate samples were evaluated under UV-vis irradiation in the presence of $5 \mathrm{mM}$ $\mathrm{Na}_{2} \mathrm{~S}_{2} \mathrm{O}_{8}$ as a sacrificial agent (Figure $2 \mathrm{~b}$ ). A set of four cycles, each with a $3 \mathrm{~h}$ duration, was performed. The Niobate/PB hybrid sample exhibits an enhanced water oxidation photocatalytic activity $\left(89.5 \mu \mathrm{mol} \mathrm{g}{ }^{-1} \mathrm{~h}^{-1}\right)$ in the first $1 \mathrm{~h}$ of each cycle, which is ca. 6.5 times higher than that of $\mathrm{KCa}_{2} \mathrm{Nb}_{3} \mathrm{O}_{10}$ 
(13.4 $\mu \mathrm{mol} \mathrm{g}^{-1} \mathrm{~h}^{-1}$ ). During all four cycles, the activity decreased gradually after the first $1 \mathrm{~h}$, which could be attributed to the consumption of the electron scavenger. Before each cycle, $\mathrm{S}_{2} \mathrm{O}_{8}{ }^{2-}$ was added to the suspension. The similarity of all cycles suggests that the hybrid assembly maintains its activity and stability during a $12 \mathrm{~h}$ experiment. This enhancement could be attributed to a more effective charge separation process in comparison to the bare niobate sample in addition to the large surface area provided by the exfoliation-restacking route, which maintains its activity even during the fourth cycle. The photocatalytic activity of restacked $\mathrm{KCa}_{2} \mathrm{Nb}_{3} \mathrm{O}_{10}$ sheets has previously been reported to be 14 $\mu \mathrm{mol} \mathrm{g}{ }^{-1} \mathrm{~h}^{-1}$ in the presence of $\mathrm{IO}_{3}^{-}$ions. $\mathrm{RuO}_{2}$ loading was realized as an effective method for promoting water oxidation activity $\left(126 \mu \mathrm{mol} \mathrm{g}^{-1} \mathrm{~h}^{-1}\right){ }^{30} \mathrm{Pt}$ intercalation could also improve the photocatalytic activity of calcium niobate layers $\left(75 \mu \mathrm{mol} \mathrm{g}^{-1} \mathrm{~h}^{-1}\right) .^{13}$ Hybrid assemblies incorporating earthabundant catalysts, however, exhibit much lower activities. For example, the photocatalytic activity of niobate nanosheets was reduced to $10 \mu \mathrm{mol} \mathrm{g}{ }^{-1} \mathrm{~h}^{-1}$ when they were coupled with $\mathrm{CoO}_{x}$ (Table S1). ${ }^{29}$ Photocatalytic experiments with a $420 \mathrm{~nm}$ cutoff filter were also performed to determine the activity of the bare niobate and the Niobate/PB sample under visible light illumination (Figure S5). While bare niobate nanosheets are not active under visible light irradiation, an activity of 48.6 $\mu \mathrm{mol} \mathrm{g} \mathrm{g}^{-1} \mathrm{~h}^{-1}$, although around 2 times lower in comparison to the "no filter" case, is observed with the niobate/PB sample. The lower activity under visible light irradiation suggests that the hybrid assembly is active in both the UV and visible regions. CoFe-PB thus serves not only as a water oxidation catalyst but also as a visible-light absorber in the hybrid sample.

Photoelectrochemical (PEC) experiments were carried out to understand the charge transfer process and the electronhole separation efficiency of bare niobate and hybrid samples (Figure 3a). The enhanced photocurrent response of Niobate/ $\mathrm{PB}$ in comparison to $\mathrm{KCa}_{2} \mathrm{Nb}_{3} \mathrm{O}_{10}$ suggests a much higher migration and separation rate of photogenerated electrons and holes under UV-vis light illumination. The Mott-Schottky plots of $\mathrm{KCa}_{2} \mathrm{Nb}_{3} \mathrm{O}_{10}$ and Niobate/PB electrodes were also obtained, as shown in Figures $3 \mathrm{~b}, \mathrm{c}$. The flat band position of the samples was determined with eq $1^{44}$

$$
\frac{1}{C^{2}}=\frac{2}{\varepsilon_{0} \varepsilon_{\mathrm{r}} e N_{\mathrm{D}}}\left[V-V_{\mathrm{FB}}-\frac{k_{\mathrm{B}} T}{e}\right]
$$

where $C$ is the interfacial capacitance, $\varepsilon_{0}$ is the vacuum permittivity, $\varepsilon_{\mathrm{r}}$ is the dielectric constant of the semiconductor, $N_{\mathrm{D}}$ is the carrier density, $V$ is the applied voltage, $k_{\mathrm{B}}$ is Boltzmann's constant, $T$ is the absolute temperature, and $e$ is the electronic charge. The interception point of the linear region of $1 / C^{2}$ vs the applied potential $(V)$ curve with the horizontal axis corresponds to $V_{\mathrm{FB}}$. The positive slope obtained for the $\mathrm{KCa}_{2} \mathrm{Nb}_{3} \mathrm{O}_{10}$ electrode reveals that the niobate nanosheets are n-type semiconductors. The flat band potential of niobate nanosheets is estimated as $-0.6 \mathrm{~V}$ (vs NHE), which is consistent with a previous report (Figure $3 \mathrm{~b}) .{ }^{45} \mathrm{It}$ is assumed that the conduction band edge potential of the n-type metal oxide semiconductor is $0.1-0.4 \mathrm{eV}$ more negative relative to the flat band potential. ${ }^{46}$ According to the literature, this value is assumed to be $0.4 \mathrm{eV}$ for a niobate semiconductor. Therefore, the conduction band edge for niobate nanosheets was obtained as $-1 \mathrm{~V}$ (vs NHE). ${ }^{45,47}$ As seen in Figure $3 \mathrm{c}$, the flat band potential for the niobate/PB shifted to more positive potentials, suggesting a decrease in the bending of the niobate band edges. On the other hand, the p-type semiconducting character of the PB structure is evidenced by the MottSchottky analysis of the hybrid material and a previous study. ${ }^{48}$ The reverse V-shaped Mott-Schottky plot for the Niobate/PB system is typical evidence for a $\mathrm{p}-\mathrm{n}$ junction formation, which clearly explains the increase in the photocurrent and photocatalytic water oxidation activity for the Niobate/PB sample. ${ }^{4-51}$ The formation of the $\mathrm{p}-\mathrm{n}$ junction results in a strong electric field within the space charge region and decreases the charge transfer resistance at the interface, which enhances the charge carrier dynamics at the electrode/ electrolyte interface. As seen in Figure 3d, the alignment of bands makes the hole transfer through the p-type PB beneficial for the water oxidation reaction.

Layered niobate has a proper band structure for both hydrogen and oxygen evolution reactions similar to that of various wide-band-gap metal oxide semiconductors. ${ }^{28,52}$ The bulk structure of the $2 \mathrm{D}$ layered niobate, however, is not an efficient photocatalyst in comparison to its individual nanosheets obtained after exfoliation. In addition, the long diffusion length for electron-hole pairs in bulk structures increases the probability of electron/hole recombination, which in turn decreases the photocatalytic activity of the semiconductor. ${ }^{28,53}$ Exfoliation, on the other hand, separates the metal oxide building blocks of the layered structure to its individual nanosheets, which produces a larger surface area. Exfoliated nanosheets of the layered structure also behave as a singlecrystalline structure with much shorter diffusion lengths for electron-hole pairs. ${ }^{2,53}$ Another advantage of exfoliation is that the band gap of a nanosheet is greater than that of the bulk structure as a result of the confinement effect. The increase in the water oxidation photocatalytic activity after the exfoliation/ restacking route for the Niobate/PB system can also be attributed to the higher surface area, short diffusion length for electron/hole pairs, and greater availability of reaction sites on both PB structure and individual nanosheets. In addition, the strong electric field established at the $\mathrm{p}-\mathrm{n}$ junction formed by niobate nanosheets and PB particles enhances the photocatalytic water oxidation activity. The band gap of niobate nanosheets was estimated to be $3.54 \mathrm{eV}$ by fitting the absorption data with a Tauc plot (Figure S6). ${ }^{47,54}$ A cyclic voltammetry measurement reveals the HOMO level of Prussian blue structure to be around $1.41 \mathrm{~V}$ vs NHE (Figure S7). Since the valence band of the niobate nanosheets is at a more positive potential in comparison to that of $\mathrm{PB}$, the hybrid assembly exhibits a proper band energy alignment for efficient hole transfer from the niobate nanosheets to the PB structure during the water oxidation process (Figure 3d).

An X-ray photoelectron spectroscopy (XPS) analysis was performed to study the chemical composition of the Niobate/ PB sample. The Nb spectrum for the pristine sample exhibits two peaks at binding energies of 207.4 and $210.1 \mathrm{eV}$ corresponding to $\mathrm{Nb} 3 \mathrm{~d}_{5 / 2}$ and $3 \mathrm{~d}_{3 / 2}$, respectively (Figure $\mathrm{S} 8 \mathrm{a}$ ). The Ca $2 \mathrm{p}$ spectrum is deconvoluted to two strong peaks at 347.3 and $350.8 \mathrm{eV}$, which are assigned to $\mathrm{Ca} 2 \mathrm{p}_{3 / 2}$ and $\mathrm{Ca}$ $2 \mathrm{p}_{1 / 2}$ binding energies, respectively (Figure $\mathrm{S} 8 \mathrm{~b}$ ). The $\mathrm{O} 1 \mathrm{~s}$ region displays a strong peak at a binding energy of $530.4 \mathrm{eV}$ corresponding to the lattice oxygen. At higher binding energies two additional peaks at 531.8 and $532.9 \mathrm{eV}$ are observed, which can be attributed to the surface-adsorbed hydroxyl and carbonate groups (Figure S8c). ${ }^{55,56}$ The Fe $2 \mathrm{p}$ spectrum reveals the presence of both +2 and +3 oxidation states in the 

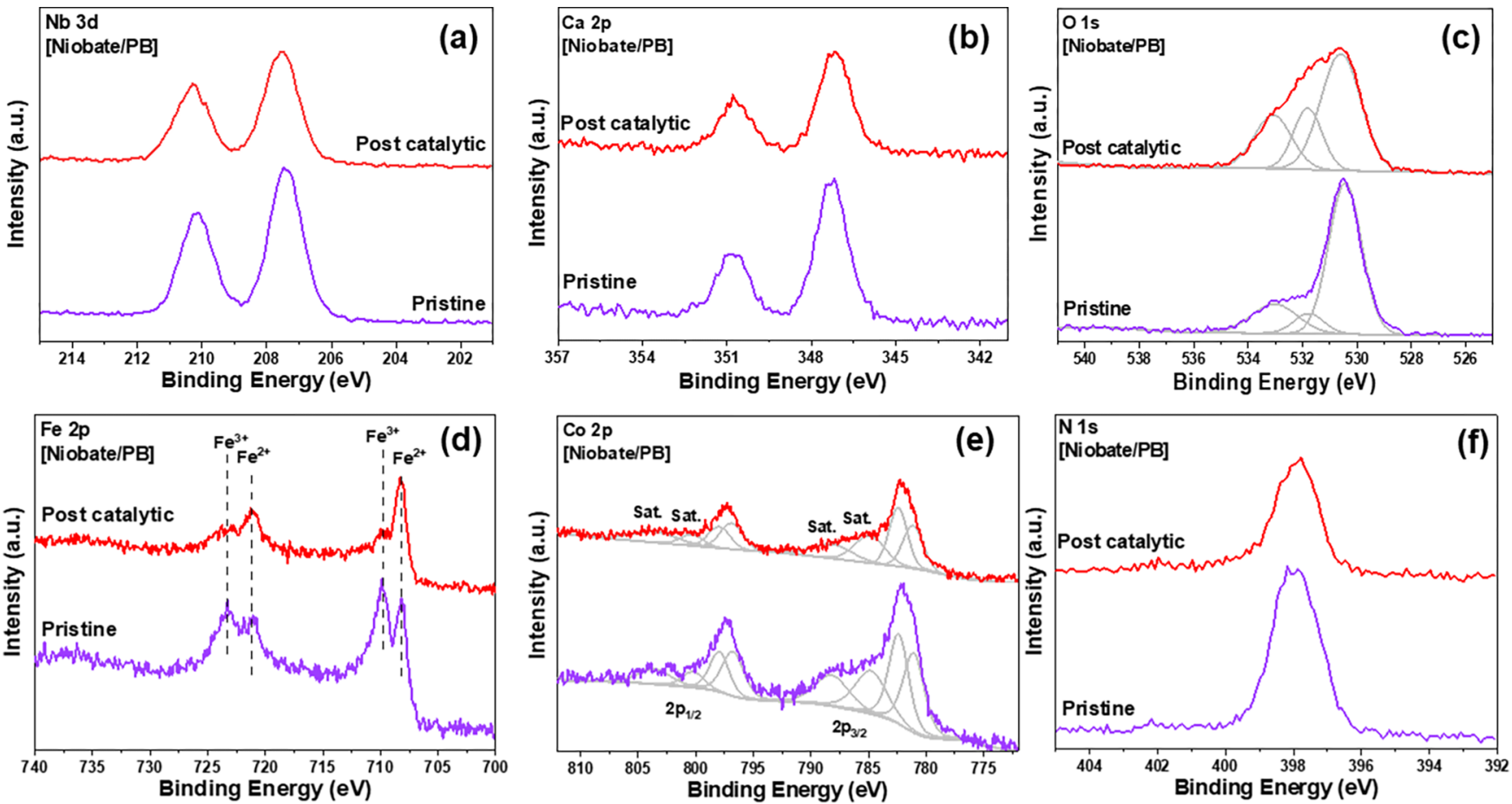

Figure 4. XPS spectra of (a) Nb 3d, (b) Ca 2p, (c) O 1s, (d) Fe 2p, (e) Co 2p, and (f) N 1s for pristine (purple line) and postcatalytic (red line) Niobate/PB samples.

hybrid sample. The peaks at 708.2 and $721.2 \mathrm{eV}$ are assigned to $\mathrm{Fe}^{2+} 2 \mathrm{p}_{3 / 2}$ and $\mathrm{Fe}^{2+} 2 \mathrm{p}_{1 / 2}$, respectively. Another doublet with slightly higher binding energies can be ascribed to $2 \mathrm{p}_{3 / 2}(709.9$ $\mathrm{eV}$ ) and $2 \mathrm{p}_{1 / 2}(723.4 \mathrm{eV})$ signals of the $\mathrm{Fe}^{3+}$ cation (Figure S8d). ${ }^{57}$ The Co $2 \mathrm{p}_{3 / 2}$ and Co $2 \mathrm{p}_{1 / 2}$ signals are deconvoluted into two main components in addition to shakeup satellite peaks at higher binding energies. The appearance of these satellites confirms the presence of $\mathrm{Co}^{2+}$ cations in the $\mathrm{PB}$ structure. $^{58-60}$ In general, the binding energy differences between $2 \mathrm{p}_{1 / 2}$ and $2 \mathrm{p}_{3 / 2}$ peaks $\left(\Delta E=2 \mathrm{p}_{1 / 2}-2 \mathrm{p}_{3 / 2}\right)$ are 15 and $16 \mathrm{eV}$ for diamagnetic $\mathrm{Co}^{\mathrm{III}}$ and paramagnetic $\mathrm{Co}^{\mathrm{II}}$ complexes, respectively. ${ }^{61-63}$ The presence of $\mathrm{Co}^{\text {III }}$ in the Niobate/PB sample is supported by $\Delta E=15.4 \mathrm{eV}$, which is intermediate between the values of $\mathrm{Co}^{\mathrm{II}}$ and $\mathrm{Co}^{\mathrm{III}}$ complexes (Figure S8e). ${ }^{64}$ The copresence of +2 and +3 oxidation states for cobalt cations is also consistent with the FTIR results of Niobate/PB sample.

A comparison of XPS profiles of Niobate/PB sample before and after the photocatalytic reaction reveals that the $\mathrm{Nb} 3 \mathrm{~d}, \mathrm{Ca}$ $2 p$, and $\mathrm{N} 1 \mathrm{~s}$ peaks remain unchanged during the photocatalytic experiment (Figure 4). As shown in Figure 4c, the peaks at 531.8 and $532.9 \mathrm{eV}$ in the postcatalytic sample are more intense, indicating the higher content of hydroxyl and carbonate groups on the surface. ${ }^{56,65}$ The amount of $\mathrm{Fe}^{2+}$ cations in the $\mathrm{PB}$ structure increases during the photocatalytic reaction (Figure $4 \mathrm{~d}$ ). The reduced intensities of Co $2 \mathrm{p}_{3 / 2}$ and $2 \mathrm{p}_{1 / 2}$ satellites and the lower value of spin-orbit splitting $(\Delta E$ $=15.2 \mathrm{eV}$ ) in the postcatalytic sample in comparison to the pristine sample support the thesis that the $\mathrm{Co}^{2+}$ ions are partially oxidized to higher oxidation states during the photocatalytic process (Figure 4e).$^{58,63}$ These results are also in agreement with postcatalytic FTIR of the Niobate/PB sample, where the intensity of the band at $2160 \mathrm{~cm}^{-1}$ (Co(II)-NC-Fe(III) coordination mode) decreases compared to that at $2110 \mathrm{~cm}^{-1}(\mathrm{Co}(\mathrm{III})-\mathrm{NC}-\mathrm{Fe}(\mathrm{II})$ coordination mode; Figure S10). The stability of the hybrid assembly is also confirmed by an SEM analysis of the postcatalytic sample (Figure S11).

\section{CONCLUSIONS}

A CoFe Prussian blue analogue was prepared in situ on niobate nanosheets to afford a semiconductor/catalyst hybrid assembly. The assembly is composed of niobate nanosheets and Prussian blue nanostructures with a particle size of around $50 \mathrm{~nm}$. Photocatalytic water oxidation studies were performed on both bare niobate nanosheets and the hybrid assembly in the presence of an electron scavenger, $\mathrm{S}_{2} \mathrm{O}_{8}{ }^{2-}$, to reveal that the hybrid assembly exhibits an enhanced activity throughout a $12 \mathrm{~h}$ photocatalytic experiment. Characterization studies on the postcatalytic sample suggest that not only does the hybrid assembly retains its structural integrity but also there is an electron transfer between the niobate and Prussian blue components due to charge transfer during the photocatalytic process.

In conclusion, the hybrid assembly represents one of the first efficient $\mathrm{p}-\mathrm{n}$ junction type niobate hybrid assemblies with an earth-abundant cocatalyst, since it exhibits a catalytic activity around 6.5 times higher than that of a bare niobate sample. The $\mathrm{p}-\mathrm{n}$ junction feature allows the hybrid assembly to be active in the visible region as well as the UV region. Easy structural tuning of both the niobate nanosheets and Prussian blue particles with metal doping allows several facile strategies to explore the niobate/ $\mathrm{PB}$ interaction further, which are currently under investigation.

\section{ASSOCIATED CONTENT}

\section{Supporting Information}

The Supporting Information is available free of charge at https://pubs.acs.org/doi/10.1021/acsaem.1c02188. 
TEM images, SEM images, EDS analysis, ATR-FTIR spectra, XPS spectra, and CV measurement of samples (PDF)

\section{AUTHOR INFORMATION}

\section{Corresponding Authors}

Ferdi Karadas - Department of Chemistry, Faculty of Science, Bilkent University, 06800 Ankara, Turkey; UNAM National Nanotechnology Research Center, Institute of Materials Science and Nanotechnology, Bilkent University, 06800 Ankara, Turkey; Email: karadas@fen.bilkent.edu.tr Ugur Unal - Department of Chemistry, Koç University, Istanbul 34450, Turkey; Koç University Surface Science and Technology Center (KUYTAM), Istanbul 34450, Turkey; Koc University Tupras Energy Center (KUTEM), Istanbul 34450, Turkey; ○ orcid.org/0000-0003-4718-1243; Email: ugunal@ku.edu.tr

Author

Sina Sadigh Akbari - Department of Chemistry, Faculty of Science, Bilkent University, 06800 Ankara, Turkey

Complete contact information is available at: https://pubs.acs.org/10.1021/acsaem.1c02188

\section{Notes}

The authors declare no competing financial interest.

\section{ACKNOWLEDGMENTS}

S.S.A. thanks TUBITAK for support (Project No. 118Z277).

\section{REFERENCES}

(1) Di, J.; Xiong, J.; Li, H.; Liu, Z. Ultrathin 2D Photocatalysts: Electronic-Structure Tailoring, Hybridization, and Applications. Adv. Mater. 2018, 30, 1704548.

(2) Kumar, A.; Kumar, A.; Krishnan, V. Perovskite Oxide Based Materials for Energy and Environment-Oriented Photocatalysis. ACS Catal. 2020, 10, 10253-10315.

(3) Bizeto, M. A.; Shiguihara, A. L.; Constantino, V. R. L. Layered Niobate Nanosheets: Building Blocks for Advanced Materials Assembly. J. Mater. Chem. 2009, 19, 2512.

(4) Okamoto, Y.; Ida, S.; Hyodo, J.; Hagiwara, H.; Ishihara, T. Synthesis and Photocatalytic Activity of Rhodium-Doped Calcium Niobate Nanosheets for Hydrogen Production from a Water/ Methanol System without Cocatalyst Loading. J. Am. Chem. Soc. 2011, 133, 18034-18037.

(5) Karim, M. R.; Shinoda, H.; Nakai, M.; Hatakeyama, K.; Kamihata, H.; Matsui, T.; Taniguchi, T.; Koinuma, M.; Kuroiwa, K.; Kurmoo, M.; Matsumoto, Y.; Hayami, S. Electrical Conductivity and Ferromagnetism in a Reduced Graphene-Metal Oxide Hybrid. Adv. Funct. Mater. 2013, 23, 323-332.

(6) Rabin, N. N.; Ida, S.; Karim, M. R.; Islam, M. S.; Ohtani, R.; Nakamura, M.; Koinuma, M.; Lindoy, L. F.; Hayami, S. Super Dielectric Materials of Two-Dimensional $\mathrm{TiO}_{2}$ or $\mathrm{Ca}_{2} \mathrm{Nb}_{3} \mathrm{O}_{10}$ Nanosheet Hybrids with Reduced Graphene Oxide. ACS Omega 2018, 3, 2074-2083.

(7) Sarac, F. E.; Yilmaz, C.; Acar, F. Y.; Unal, U. CdTe Quantum Dot Sensitized Hexaniobate Nanoscrolls and Their Photoelectrochemical Properties. RSC Adv. 2012, 2, 10182-10184.

(8) Morris, N. D.; Mallouk, T. E. A High-Throughput Optical Screening Method for the Optimization of Colloidal Water Oxidation Catalysts. J. Am. Chem. Soc. 2002, 124, 11114-11121.

(9) Hara, M.; Waraksa, C. C.; Lean, J. T.; Lewis, B. A.; Mallouk, T. E. Photocatalytic Water Oxidation in a Buffered Tris(2,2'Bipyridyl)Ruthenium Complex-Colloidal $\mathrm{IrO}_{2}$ System. J. Phys. Chem. A 2000, 104, 5275-5280.
(10) Ebina, Y.; Sakai, N.; Sasaki, T. Photocatalyst of Lamellar Aggregates of $\mathrm{RuO}_{\mathrm{x}}$-Loaded Perovskite Nanosheets for Overall Water Splitting. J. Phys. Chem. B 2005, 109, 17212-17216.

(11) Youngblood, W. J.; Lee, S.-H. A.; Maeda, K.; Mallouk, T. E. Visible Light Water Splitting Using Dye-Sensitized Oxide Semiconductors. Acc. Chem. Res. 2009, 42, 1966-1973.

(12) Oshima, T.; Ishitani, O.; Maeda, K. Non-Sacrificial Water Photo-Oxidation Activity of Lamellar Calcium Niobate Induced by Exfoliation. Adv. Mater. Interfaces 2014, 1, 1400131.

(13) Oshima, T.; Lu, D.; Ishitani, O.; Maeda, K. Intercalation of Highly Dispersed Metal Nanoclusters into a Layered Metal Oxide for Photocatalytic Overall Water Splitting. Angew. Chem., Int. Ed. 2015, 54, 2698-2702.

(14) Oshima, T.; Wang, Y.; Lu, D.; Yokoi, T.; Maeda, K. Photocatalytic Overall Water Splitting on Pt Nanocluster-Intercalated, Restacked $\mathrm{KCa}_{2} \mathrm{Nb}_{3} \mathrm{O}_{10}$ Nanosheets: The Promotional Effect of CoExisting Ions. Nanoscale Adv. 2019, 1, 189-194.

(15) Pintado, S.; Goberna-Ferrón, S.; Escudero-Adán, E. C.; GalánMascarós, J. R. Fast and Persistent Electrocatalytic Water Oxidation by Co-Fe Prussian Blue Coordination Polymers. J. Am. Chem. Soc. 2013, 135, 13270-13273.

(16) Goberna-Ferron, S.; Hernandez, W. Y.; Rodriguez-Garcia, B.; Galan-Mascaros, J. R. Light-Driven Water Oxidation with Metal Hexacyanometallate Heterogeneous Catalysts. ACS Catal. 2014, 4, 1637-1641.

(17) Aksoy, M.; Nune, S. V. K.; Karadas, F. A Novel Synthetic Route for the Preparation of an Amorphous $\mathrm{Co} / \mathrm{Fe}$ Prussian Blue Coordination Compound with High Electrocatalytic Water Oxidation Activity. Inorg. Chem. 2016, 55, 4301-4307.

(18) Alsaç, E. P.; Ülker, E.; Nune, S. V. K.; Dede, Y.; Karadas, F. Tuning the Electronic Properties of Prussian Blue Analogues for Efficient Water Oxidation Electrocatalysis: Experimental and Computational Studies. Chem. - Eur. J. 2018, 24, 4856-4863.

(19) Han, L.; Tang, P.; Reyes-Carmona, Á.; Rodríguez-García, B.; Torréns, M.; Morante, J. R.; Arbiol, J.; Galan-Mascaros, J. R. Enhanced Activity and Acid pH Stability of Prussian Blue-Type Oxygen Evolution Electrocatalysts Processed by Chemical Etching. J. Am. Chem. Soc. 2016, 138, 16037-16045.

(20) Yamada, Y.; Oyama, K.; Gates, R.; Fukuzumi, S. High Catalytic Activity of Heteropolynuclear Cyanide Complexes Containing Cobalt and Platinum Ions: Visible-Light Driven Water Oxidation. Angew. Chem., Int. Ed. 2015, 54, 5613-5617.

(21) Aratani, Y.; Oyama, K.; Suenobu, T.; Yamada, Y.; Fukuzumi, S. Photocatalytic Hydroxylation of Benzene by Dioxygen to Phenol with a Cyano-Bridged Complex Containing FeII and RuII Incorporated in Mesoporous Silica-Alumina. Inorg. Chem. 2016, 55, 5780-5786.

(22) Hegner, F. S.; Herraiz-Cardona, I.; Cardenas-Morcoso, D.; López, N.; Galán-Mascarós, J. R.; Gimenez, S. Cobalt Hexacyanoferrate on BiVO4 Photoanodes for Robust Water Splitting. ACS Appl. Mater. Interfaces 2017, 9, 37671-37681.

(23) Yamada, Y.; Oyama, K.; Suenobu, T.; Fukuzumi, S. Photocatalytic Water Oxidation by Persulphate with a $\mathrm{Ca}^{2+}$ IonIncorporated Polymeric Cobalt Cyanide Complex Affording $\mathrm{O}_{2}$ with 200\% Quantum Efficiency. Chem. Commun. 2017, 53, 34183421.

(24) Hegner, F. S.; Cardenas-Morcoso, D.; Giménez, S.; López, N.; Galan-Mascaros, J. R. Level Alignment as Descriptor for Semiconductor/Catalyst Systems in Water Splitting: The Case of Hematite/Cobalt Hexacyanoferrate Photoanodes. ChemSusChem 2017, 10, 4552-4560.

(25) Fukuzumi, S.; Jung, J.; Yamada, Y.; Kojima, T.; Nam, W. Homogeneous and Heterogeneous Photocatalytic Water Oxidation by Persulfate. Chem. - Asian J. 2016, 11, 1138-1150.

(26) Ulusoy Ghobadi, T. G.; Ozbay, E.; Karadas, F. How to Build Prussian Blue-Based Water Oxidation Catalytic Assemblies? Common Trends and Strategies. Chem. - Eur. J. 2021, 27, 3638.

(27) Maeda, K.; Eguchi, M.; Oshima, T. Perovskite Oxide Nanosheets with Tunable Band-Edge Potentials and High Photo- 
catalytic Hydrogen-Evolution Activity. Angew. Chem., Int. Ed. 2014, 53, 13164-13168.

(28) Maeda, K.; Mallouk, T. E. Two-Dimensional Metal Oxide Nanosheets as Building Blocks for Artificial Photosynthetic Assemblies. Bull. Chem. Soc. Jpn. 2019, 92, 38-54.

(29) Oshima, T.; Eguchi, M.; Maeda, K. Photocatalytic Water Oxidation over Metal Oxide Nanosheets Having a Three-Layer Perovskite Structure. ChemSusChem 2016, 9, 396-402.

(30) Oshima, T.; Ishitani, O.; Maeda, K. Non-Sacrificial Water Photo-Oxidation Activity of Lamellar Calcium Niobate Induced by Exfoliation. Adv. Mater. Interfaces 2014, 1, 1400131.

(31) Maeda, K.; Eguchi, M.; Youngblood, J. W.; Mallouk, T. E. Calcium Niobate Nanosheets Prepared by the Polymerized Complex Method as Catalytic Materials for Photochemical Hydrogen Evolution. Chem. Mater. 2009, 21, 3611-3617.

(32) Ulusoy Ghobadi, T. G.; Akhuseyin Yildiz, E.; Buyuktemiz, M.; Sadigh Akbari, S.; Topkaya, D.; İsci, Ü.; Dede, Y.; Yaglioglu, H. G.; Karadas, F. A Noble-Metal-Free Heterogeneous PhotosensitizerRelay-Catalyst Triad Catalyzes Water Oxidation under Visible Light. Angew. Chem., Int. Ed. 2018, 57, 17173-17177.

(33) Ulusoy Ghobadi, T. G.; Ghobadi, A.; Buyuktemiz, M.; Yildiz, E. A.; Berna Yildiz, D.; Yaglioglu, H. G.; Dede, Y.; Ozbay, E.; Karadas, F. A Robust, Precious-Metal-Free Dye-Sensitized Photoanode for Water Oxidation: A Nanosecond-Long Excited-State Lifetime through a Prussian Blue Analogue. Angew. Chem., Int. Ed. 2020, 59, 4082-4090.

(34) Kap, Z.; Karadas, F. Visible Light-Driven Water Oxidation with a Ruthenium Sensitizer and a Cobalt-Based Catalyst Connected with a Polymeric Platform. Faraday Discuss. 2019, 215, 111-122.

(35) Kap, Z.; Ülker, E.; Nune, S. V. K.; Karadas, F. Electrocatalytic Hydrogen Evolution with Cobalt-Poly(4-Vinylpyridine) Metallopolymers. J. Appl. Electrochem. 2018, 48, 201-209.

(36) Akbari, S. S.; Karadas, F. Precious Metal-Free Photocatalytic Water Oxidation by a Layered Double Hydroxide-Prussian Blue Analogue Hybrid Assembly. ChemSusChem 2021, 14, 679-685.

(37) Gundogdu, G.; Ulusoy Ghobadi, T. G.; Sadigh Akbari, S.; Ozbay, E.; Karadas, F. Photocatalytic Water Oxidation with a Prussian Blue Modified Brown $\mathrm{TiO}_{2}$. Chem. Commun. 2021, 57, 508-511.

(38) Hata, H.; Kobayashi, Y.; Bojan, V.; Youngblood, W. J.; Mallouk, T. E. Direct Deposition of Trivalent Rhodium Hydroxide Nanoparticles onto a Semiconducting Layered Calcium Niobate for Photocatalytic Hydrogen Evolution. Nano Lett. 2008, 8, 794-799.

(39) Wang, Q.-Q.; Lin, B.-Z.; Xu, B.-H.; Li, X.-L.; Chen, Z.-J.; Pian, X.-T. Preparation and Photocatalytic Properties of Mesoporous $\mathrm{SnO}_{2}-$ Hexaniobate Layered Nanocomposite. Microporous Mesoporous Mater. 2010, 130, 344-351.

(40) Kong, X.; Zeng, C.; Wang, X.; Huang, J.; Li, C.; Fei, J.; Li, J.; Feng, Q. Ti-O-O Coordination Bond Caused Visible Light Photocatalytic Property of Layered Titanium Oxide. Sci. Rep. 2016, 6, 29049.

(41) Gerber, S. J.; Erasmus, E. Electronic Effects of Metal Hexacyanoferrates: An XPS and FTIR Study. Mater. Chem. Phys. 2018, 203, 73-81.

(42) Sato, O.; Iyoda, T.; Fujishima, A.; Hashimoto, K. Photoinduced Magnetization of a Cobalt-Iron Cyanide. Science 1996, 272, 704-705.

(43) Sato, O.; Einaga, V.; Iyoda, T.; Fujishima, A.; Hashimoto, K. Reversible Photoinduced Magnetization. J. Electrochem. Soc. 1997, 144, L11.

(44) Gelderman, K.; Lee, L.; Donne, S. W. Flat-Band Potential of a Semiconductor: Using the Mott-Schottky Equation. J. Chem. Educ. 2007, 84, 685-688.

(45) Xu, P.; Milstein, T. J.; Mallouk, T. E. Flat-Band Potentials of Molecularly Thin Metal Oxide Nanosheets. ACS Appl. Mater. Interfaces 2016, 8, 11539-11547.

(46) Matsumoto, Y. Energy Positions of Oxide Semiconductors and Photocatalysis with Iron Complex Oxides. J. Solid State Chem. 1996, 126, 227-234.

(47) Akatsuka, K.; Takanashi, G.; Ebina, Y.; Haga, M. A.; Sasaki, T. Electronic Band Structure of Exfoliated Titanium-and/or NiobiumBased Oxide Nanosheets Probed by Electrochemical and Photo- electrochemical Measurements. J. Phys. Chem. C 2012, 116, 1242612433.

(48) Wojdeł, J. C.; Moreira, I. de P. R.; Bromley, S. T.; Illas, F. Prediction of Half-Metallic Conductivity in Prussian Blue Derivatives. J. Mater. Chem. 2009, 19, 2032-2036.

(49) Wang, Y. C.; Chang, C. Y.; Yeh, T. F.; Lee, Y. L.; Teng, H. Formation of Internal p-n Junctions in $\mathrm{Ta}_{3} \mathrm{~N}_{5}$ Photoanodes for Water Splitting. J. Mater. Chem. A 2014, 2, 20570-20577.

(50) Wang, S.; Huang, C. Y.; Pan, L.; Chen, Y.; Zhang, X.; Fazal-eAleem; Zou, J. J. Controllable Fabrication of Homogeneous $\mathrm{ZnO}$ p-n Junction with Enhanced Charge Separation for Efficient Photocatalysis. Catal. Today 2019, 335, 151-159.

(51) Lim, H.; Kim, J. Y.; Evans, E. J.; Rai, A.; Kim, J. H.; Wygant, B. R.; Mullins, C. B. Activation of a Nickel-Based Oxygen Evolution Reaction Catalyst on a Hematite Photoanode via Incorporation of Cerium for Photoelectrochemical Water Oxidation. ACS Appl. Mater. Interfaces 2017, 9, 30654-30661.

(52) Ida, S.; Ishihara, T. Recent Progress in Two-Dimensional Oxide Photocatalysts for Water Splitting. J. Phys. Chem. Lett. 2014, 5, 25332542.

(53) Hu, Y.; Mao, L.; Guan, X.; Tucker, K. A.; Xie, H.; Wu, X.; Shi, J. Layered Perovskite Oxides and Their Derivative Nanosheets Adopting Different Modification Strategies towards Better Photocatalytic Performance of Water Splitting. Renewable Sustainable Energy Rev. 2020, 119, 109527.

(54) Compton, O. C.; Carroll, E. C.; Kim, J. Y.; Larsen, D. S.; Osterloh, F. E. Calcium Niobate Semiconductor Nanosheets as Catalysts for Photochemical Hydrogen Evolution from Water. J. Phys. Chem. C 2007, 111, 14589-14592.

(55) Naylor, A. J.; Makkos, E.; Maibach, J.; Guerrini, N.; Sobkowiak, A.; Björklund, E.; Lozano, J. G.; Menon, A. S.; Younesi, R.; Roberts, M. R.; Edström, K.; Islam, M. S.; Bruce, P. G. Depth-Dependent Oxygen Redox Activity in Lithium-Rich Layered Oxide Cathodes. J. Mater. Chem. A 2019, 7, 25355-25368.

(56) Sarac, F. E.; Unal, U. Electrochemical-Hydrothermal Synthesis of Manganese Oxide Films As Electrodes for Electrochemical Capacitors. Electrochim. Acta 2015, 178, 199-208.

(57) Cui, X.; Hong, L.; Lin, X. Electrochemical Preparation, Characterization and Application of Electrodes Modified with Hybrid Hexacyanoferrates of Copper and Cobalt. J. Electroanal. Chem. 2002, $526,115-124$.

(58) Haraguchi, H.; Fujiwara, K.; Keiichiro, F. A Study of Cobalt Complexes by X-Ray Photoelectron Spectroscopy. Chem. Lett. 1975, 4, 409-414.

(59) Atzei, D.; De Filippo, D.; Rossi, A.; Caminiti, R. X-Ray Photoelectron Spectra of Dinitrogen Chelating Ligands with Some Transition Metals. Spectrochim. Acta Part A Mol. Spectrosc. 1993, 49, $1779-1785$.

(60) Lu, K.; Song, B.; Gao, X.; Dai, H.; Zhang, J.; Ma, H. HighEnergy Cobalt Hexacyanoferrate and Carbon Micro-Spheres Aqueous Sodium-Ion Capacitors. J. Power Sources 2016, 303, 347-353.

(61) Briggs, D.; Gibson, V. A. Direct Observation of Multiplet Splitting in $2 p$ Photoelectron Peaks of Cobalt Complexes. Chem. Phys. Lett. 1974, 25, 493-496.

(62) Atzei, D.; Rossi, A.; Sadun, C. Synthesis and Characterization of a Cobalt(III) Complex with 1-(D-3-Mercapto-Methylpropionyl)-LProline. Spectrochim. Acta, Part A 2000, 56, 1875-1886.

(63) Ivanova, T.; Naumkin, A.; Sidorov, A.; Eremenko, I.; Kiskin, M. X-Ray Photoelectron Spectra and Electron Structure of Polynuclear Cobalt Complexes. J. Electron Spectrosc. Relat. Phenom. 2007, 156$158,200-203$.

(64) Berrettoni, M.; Ciabocco, M.; Fantauzzi, M.; Giorgetti, M.; Rossi, A.; Caponetti, E. Physicochemical Characterization of Metal Hexacyanometallate- $\mathrm{TiO}_{2}$ Composite Materials. RSC Adv. 2015, 5, 35435-35447.

(65) Hekmatfar, M.; Kazzazi, A.; Eshetu, G. G.; Hasa, I.; Passerini, S. Understanding the Electrode/Electrolyte Interface Layer on the LiRich Nickel Manganese Cobalt Layered Oxide Cathode by XPS. ACS Appl. Mater. Interfaces 2019, 11, 43166-43179. 